\title{
Symmetry in Concurrent Games
}

\author{
Simon Castellan \\ Ecole Normale Supérieure de Lyon, France
}

\author{
Pierre Clairambault Glynn Winskel \\ Computer Laboratory, University of Cambridge, UK
}

\begin{abstract}
Behavioural symmetry is introduced into concurrent games. It expresses when plays are essentially the same. A characterization of strategies on games with symmetry is provided. This leads to a bicategory of strategies on games with symmetry. Symmetry helps allay the perhaps overly-concrete nature of games and strategies, and shares many mathematical features with homotopy. In the presence of symmetry we can consider monads for which the monad laws do not hold on the nose but do hold up to symmetry. This broadening of the concept of monad has a dramatic effect on the types concurrent games can support and allows us, for example, to recover the replication needed to express and extend traditional game semantics of programming languages.
\end{abstract}

\section{INTRODUCTION}

In game semantics of programming languages a type of a program is represented by a game, and the program itself by a strategy in the game. The approach is very flexible: game semantics has managed to capture accurately a wide variety of features of higher-order programming languages, including state, control, exceptions, and many others. Game semantics follows the methodology of denotational semantics, and interprets complex programs compositionally in terms of interpretations of their components. Game semantics is also operational: moves in a game correspond to computation steps. As such it is increasingly exploited to provide a syntax-free operational semantics for programming languages, a much needed tool for the analysis and verification of programs.

Concurrency is a central concern in computer science. Reflecting the traditions of concurrency theory, game semantics for concurrent programs come in two styles: based on interleavings and on partial-orders. Of these, the historical focus has been on interleaving, giving models of various programming languages [1], [2], even a model-checking tool [3]. Partial-order methods have the strength of supporting reasoning about dependency directly, but are at a more preliminary stage. Abramsky and Melliès proposed in [4] a framework for concurrent games based on closure operators, re-understood later by Melliès and Mimram in terms of asynchronous transition systems [5], [6]; Faggian and Piccolo have also presented strategies as partial orders [7]. In [8], Rideau and Winskel gave a new foundation based on event structures, generalizing all previous approaches and allowing nondeterminism. It is this framework we refer to as concurrent games.

When developing a game semantics, one has to deal with the low-level aspect of games-some identities that hold operationally do not hold automatically in games. An important example of such phenomena occurs in the replication of resources in programming languages: whereas two accesses to the same resource might be indistinguishable operationally, they can correspond to different and unrelated events in the game. In this sense, games are overly-concrete. In the history of game semantics [9], this has been alleviated by introducing symmetry into games. Informally, symmetry in a game concerns when one play of a game is essentially the same as another. Our treatment of symmetry in concurrent games, where plays can be highly-distributed, stems from earlier work on symmetry in event structures [10] and makes use of a general method of open maps for defining bisimulation in a variety of models [11]. Briefly, a symmetry in a game is expressed as a bisimulation equivalence (given as a span of open maps) that says when two plays are similar according to the symmetry. This feature considerably enhances the mathematical theory of concurrent games. Symmetry comes to share many features with homotopy-symmetric plays are like homotopic paths-which plays a role in its mathematical development.

a) Contributions: Firstly, we introduce concurrent games with symmetry. This involves a new definition of the copycat strategy and of composition, which now have to respect symmetry. As in [8], we characterize strategies, for which copycat behaves like an identity w.r.t. composition. This leads to the construction of a bicategory (up to symmetry) of concurrent games with symmetry and symmetry-respecting strategies. Secondly, we give two illustrations of how this framework can be used to model logics and programming languages. The first is a presentation within concurrent games of the construction of [12], using an adaptation of AJM games [9] to model classical linear logic. The second is a concurrent games presentation of $\mathrm{HO}$ games [13], giving a concurrent and non-deterministic notion of innocent strategies (in the sense of Hyland and Ong), and a new proof that standard innocent strategies are stable under composition.

b) Related work: In sequential games, the notion of symmetry that is closest to ours is that of AJM games [9], and in particular its variant in [12]. In asynchronous games, Melliès expressed symmetry by giving groups acting on the game, reindexing the events. Restricting to a polarized deterministic setting, it should be possible to reformulate Melliès' approach in terms of concurrent games with symmetry-left however for future work.

c) Outline: In Section II, we present event structures, and their extension with symmetry and polarity. In Section III, we give the main contribution of this paper, the bicategory of concurrent games with symmetry and concurrent strategies. 
In Section IV we give the two applications: the concurrent generalization of (a variant of) AJM games and $\mathrm{HO}$ games.

\section{PRELIMINARIES}

\section{A. Event structures}

An event structure comprises $(E, \leq$, Con $)$, consisting of a set $E$, of events which are partially ordered by $\leq$, the causal dependency relation, and a nonempty consistency relation Con consisting of finite subsets of $E$, which satisfy

$$
\begin{aligned}
& \left\{e^{\prime} \mid e^{\prime} \leq e\right\} \text { is finite for all } e \in E, \\
& \{e\} \in \text { Con for all } e \in E, \\
& Y \subseteq X \in \text { Con } \Longrightarrow Y \in \text { Con, and } \\
& X \in \text { Con } \& e \leq e^{\prime} \in X \Longrightarrow X \cup\{e\} \in \text { Con. }
\end{aligned}
$$

The (finite) configurations, $\mathcal{C}(E)$, of an event structure $E$ consist of those finite subsets $x \subseteq E$ which are

Consistent: $x \in$ Con, and

Down-closed: $\forall e, e^{\prime} . e^{\prime} \leq e \in x \Longrightarrow e^{\prime} \in x$.

We say an event structure is elementary when the consistency relation consists of all finite subsets of events. For $X \subseteq E$ we write $[X]_{E}$ for $\left\{e \in E \mid \exists e^{\prime} \in X . e \leq e^{\prime}\right\}$, the down-closure of $X$; note if $X \in$ Con, then $[X]_{E} \in$ Con is a configuration. In games the relation of immediate dependency $e \rightarrow e^{\prime}$, meaning $e$ and $e^{\prime}$ are distinct with $e \leq e^{\prime}$ and no event in between, will play a very important role. For configurations $x, y$, we use $x-\subset y$ to mean $y$ covers $x$, i.e. $x \subset y$ with nothing in between, and $x \stackrel{e}{-} \subset y$ to mean $x \cup\{e\}=y$ for $x, y \in \mathcal{F}$ and event $e \notin x$. We sometimes use $x \stackrel{e}{-}$, expressing that event $e$ is enabled at configuration $x$, when $x \stackrel{e}{-} c y$ for some $y$.

A (partial) map of event structures $f: E \rightarrow E^{\prime}$ is a partial function $f: E \rightarrow E^{\prime}$ from the events of $E$ to the events of $E^{\prime}$ such that for all configurations $x \in \mathcal{C}(E)$,

$$
f x \in \mathcal{C}\left(E^{\prime}\right) \&\left(\forall e_{1}, e_{2} \in x . f\left(e_{1}\right)=f\left(e_{2}\right) \Longrightarrow e_{1}=e_{2}\right) .
$$

Maps compose as functions. We say a map is total when it is total as a function; then $f$ restricts to a bijection $x \cong f x$ on $x \in \mathcal{C}(E)$. Say a total map of event structures is rigid when it preserves causal dependency. We write $\mathcal{E}$ for the category of event structures with total maps, and $\mathcal{E}_{r}$ and $\mathcal{E}_{p}$ for the categories with rigid and partial maps, respectively. All three categories have coproducts, binary products and pullbacks. The inclusion functor $\mathcal{E}_{r} \hookrightarrow \mathcal{E}$ has a right adjoint, in fact so that $\mathcal{E}$ is the Kleisli category of the monad for the adjunction [10]. A right adjoint to the inclusion $\mathcal{E} \hookrightarrow \mathcal{E}_{p}$ will have to await the introduction of symmetry.

Proposition 1. Finite configurations of a product $A \times B$ in $\mathcal{E}$ correspond to secured bijections $\theta: x \cong y$ between configurations $x \in \mathcal{C}(A)$ and $y \in \mathcal{C}(B)$, such that the order generated on $\theta$ by taking $(a, b) \leq\left(a^{\prime}, b^{\prime}\right)$ if $a \leq_{A}$ a or $b \leq_{B} b^{\prime}$ is a partial order. The correspondence respects inclusion.

Individual configurations inherit an order from the ambient event structure and can themselves be regarded as finite elementary event structures. Viewed this way, an inclusion $x \subseteq y$ between configurations induces a rigid map $x \hookrightarrow y$ between the configurations regarded as event structures. The configurations of an event structure form a non-empty family of finite partial orders closed under rigid inclusions. Conversely, given such a rigid family, we can build an event structure:

Proposition 2. Let $\mathcal{Q}$ be a rigid family, a non-empty family of finite partial orders closed under rigid inclusions, i.e. if $q \in \mathcal{Q}$ and $q^{\prime} \hookrightarrow q$ is a rigid inclusion (regarded as a map of event structures) then $q^{\prime} \in \mathcal{Q}$. The family $\mathcal{Q}$ determines an event structure $\operatorname{Pr}(\mathcal{Q})={ }_{\mathrm{def}}(P, \leq$, Con $)$ as follows:

- the events $P$ are the prime partial orders in $\mathcal{Q}$, i.e. those finite partial orders in $\mathcal{Q}$ with a top element;

- the causal dependency relation $p^{\prime} \leq p$ holds precisely when there is a rigid inclusion from $p^{\prime} \leftrightarrow p$;

- a finite subset $X \subseteq P$ is consistent, $X \in$ Con, iff there is $q \in \mathcal{Q}$ and rigid inclusions $p \hookrightarrow q$ for all $p \in X$.

If $x \in \mathcal{C}(P)$ then $\cup x$, the union of the partial orders in $x$, is in $\mathcal{Q}$. The function $x \mapsto \cup x$ is an order-isomorphism from $\mathcal{C}(P)$, ordered by inclusion, to $\mathcal{Q}$, ordered by rigid inclusions.

\section{B. Symmetry}

We endow event structures with symmetry. A relation of symmetry on an event structure expresses when two configurations are essentially the same and is expressed as a form of bisimulation equivalence, based on open maps [11].

1) Open maps: Open maps are a generalisation of functional bisimulations, known from transition systems. Let $\mathcal{C}$ be a category with a distinguished subcategory $\mathcal{P}$ of path objects with path-extension maps. A map $f: A \rightarrow B$ in $\mathcal{C}$ is open if it satisfies a path-lifting property:

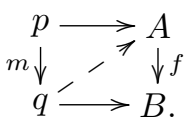

Any commuting square, with $m: p \rightarrow q$ in $\mathcal{P}$, factors into two commuting triangles as shown. Bisimulation is then expressed as a span of open maps.

W.r.t. any of the categories $\mathcal{E}, \mathcal{E}_{r}$ or $\mathcal{E}_{p}$, taking $\mathcal{P}$ to be the full subcategory of finite elementary event structures, it can be shown [10] that a map $h: A \rightarrow B$ of event structures is open iff $h$ is rigid and satisfies

$\forall x \in \mathcal{C}(A), y^{\prime} \in \mathcal{C}(B) . h x \subseteq y^{\prime} \Rightarrow \exists x^{\prime} \in \mathcal{C}(E) . x \subseteq x^{\prime} \& h x^{\prime}=y^{\prime}$.

2) Event structures with symmetry: The way we equip event structures with symmetry is an instance of the following general construction.

Let $X$ be an object of a category $\mathcal{C}$. Recall that a relation on $X$ is an object $\widetilde{X}$ and pair of maps $l_{X}, r_{X}: \widetilde{X} \rightarrow X-$ so forming a span-which are jointly-monic. A map between objects with relations $f:(X, \widetilde{X}) \rightarrow(Y, \widetilde{Y})$ is a map $f: X \rightarrow$ $Y$ in $\mathcal{C}$ for which there is a necessarily unique map $\widetilde{f}: \widetilde{X} \rightarrow \widetilde{Y}$ in $\mathcal{C}$ such that $f l_{X}=l_{Y} \widetilde{f}$ and $f r_{X}=r_{Y} \widetilde{f}$. If $\mathcal{C}$ has products, then a relation on $X$ can equivalently be given by a mono $\widetilde{X} \succ$ $X \times X$. If $\mathcal{C}$ has pullbacks we can formulate diagrammatically 
the requirement that $\widetilde{X}$ be an equivalence relation-see the Appendix.

If $\mathcal{C}$ is equipped with a class of open maps, we say that a relation $l_{X}, r_{X}: \widetilde{X} \rightarrow X$ is a symmetry in $X$ if it is an equivalence relation with both $l_{X}$ and $r_{X}$ open; this amounts to $\widetilde{X}$ being a bisimulation equivalence. A map $f:(X, \widetilde{X}) \rightarrow(Y, \widetilde{Y})$ necessarily preserves symmetry. We obtain a category $\mathcal{C S}$ of objects with symmetry and symmetrypreserving maps. Given two maps $f, g:(X, \widetilde{X}) \rightarrow(Y, \widetilde{Y})$ they are equal up to symmetry, written $f \sim g$, if there is a necessarily unique map $h: X \rightarrow \widetilde{Y}$ in $\mathcal{C}$ such that $f=l_{Y} h$ and $g=r_{Y} h$. The category $\mathcal{C S}$ is more fully described as enriched in the category of equivalence relations and so, because equivalence relations are a degenerate form of category, as a 2-category in which the 2-cells are instances of the equivalence. This view informs their constructions in such categories which are often very simple examples of the (pseudo- and bi-) constructions of 2-categories. In particular, objects $A$ and $B$ are equivalent, written $A \simeq B$, if there are maps $f: A \rightarrow B$ and $g: B \rightarrow A$ such that $f g \sim \operatorname{id}_{B}$ and $g f \sim \operatorname{id}_{A}$.

We can take advantage of the concrete nature of event structures to give an explicit description of symmetries there, a characterization which is independent of whether the category of event structures carries rigid, total or partial maps, though for definiteness assume the category is $\mathcal{E}$. A symmetry in an event structure $E$ determines a mono $\widetilde{E} \nrightarrow E \times E$, thus a subset of $\mathcal{C}(E \times E)$, and so by Proposition 1 a family of bijections between finite configurations of $E$. In this way, a symmetry in an event structure $E$ corresponds to an isomorphism family comprising a non-empty family of bijections $\theta: x \cong_{E} y$ between pairs of finite configurations of $E$ such that:

(i) for all identities $\operatorname{id}_{x}: x \cong_{E} x$, where $x \in \mathcal{C}(E)$; if $\theta: x \cong_{E} y$, then the inverse $\theta^{-1}: y \cong_{E} x$; and if $\theta: x \cong_{E} y$ and $\varphi: y \cong_{E} z$, then their composition $\varphi \circ \theta: x \cong_{E} z$.

(ii) for $\theta: x \cong_{E} y$ whenever $x^{\prime} \subseteq x$ with $x^{\prime} \in \mathcal{C}(E)$, then there is a (necessarily unique) $y^{\prime} \in \mathcal{C}(E)$ with $y^{\prime} \subseteq y$ such that the restriction $\theta^{\prime}: x^{\prime} \cong_{E} y^{\prime}$.

(iii) for $\theta: x \cong_{E} y$ whenever $x \subseteq x^{\prime}$ for $x^{\prime} \in \mathcal{C}(E)$, there is an extension of $\theta$ to $\theta^{\prime}$ so $\theta^{\prime}: x^{\prime} \cong_{E} y^{\prime}$ for some (not necessarily unique) $y^{\prime} \in \mathcal{C}(E)$ with $y \subseteq y^{\prime}$.

The isomorphism family makes precise the sense in which a symmetry expresses when two configurations are essentially the same. Note that (ii) implies that the bijections in the isomorphism family respect the partial order of causal dependency on configurations inherited from $E$; the bijections in an isomorphism family are isomorphisms between the configurations regarded as elementary event structures.

An event structure with symmetry $A$ corresponds to an isomorphism family $\cong_{A}$ of the underlying event structure of $A$ [10]. There are straightforward reformulations of what it means for a map to preserve symmetry or for two maps to be equal up to symmetry in terms of isomorphism families. A total map $f: A \rightarrow B$ preserving symmetry amounts to $x \stackrel{\theta}{\cong_{A}} y$ implying $f x \stackrel{\widetilde{f} \theta}{\cong}_{B} f y$, where $\widetilde{f} \theta$ is the composite bijection $f x \cong x \stackrel{\theta}{=} A \cong f y$; while $f \sim g$, for two total maps $f, g: A \rightarrow B$ preserving symmetry, iff $f x \stackrel{\varphi}{\cong}_{B} g x$ for all $x \in \mathcal{C}(A)$, where $\varphi_{x}$ is the composite bijection $f x \cong x \cong g x$.

We define the category $\mathcal{E S}$ to consist of event structures with symmetry, with total maps. Similarly, $\mathcal{E S}_{r}$ and $\mathcal{E S}_{p}$ are the categories sharing the same objects but with rigid and partial maps. Now both the inclusion functors $\mathcal{E S} r \rightarrow \mathcal{E S}$ and $\mathcal{E S} \rightarrow \mathcal{E S}_{p}$ have right adjoints so that $\mathcal{E S}$ and $\mathcal{E S}_{p}$ are Kleisli categories of the respective monads [10].

Proposition 3. Any map $f: A \rightarrow B$ in $\mathcal{E S}_{p}$ has a partial-total factorization as a composite

$$
A \stackrel{p_{V}}{\longrightarrow}(A \downarrow V) \stackrel{f_{1}}{\longrightarrow} B
$$

where: $V=\operatorname{def}\{a \in A \mid f(a)$ is defined $\}$ is the domain of definition of $f$; $A \downarrow V==_{\text {def }}\left(V, \leq_{V}, \operatorname{Con}_{V}\right)$ with $v \leq_{V} v^{\prime}$ iff $v \leq_{A}$ $v^{\prime} \& v, v^{\prime} \in V$ and $X \in \mathrm{Con}_{V}$ iff $X \in \mathrm{Con}_{A} \& X \subseteq V$; its isomorphism family is given by $\theta: x \cong \cong_{A \downarrow V}$ y iff $\theta$ extends to $\theta^{\prime}:[x]_{A} \cong_{A}[y]_{A}$; the map $p_{V}: A \rightarrow A \downarrow V$ is the partial map acting as identity on $V$ and undefined elsewhere; and $f_{1}$ is a total map acting as $f$ on $V$. If $f \sim g: A \rightarrow B$ in $\mathcal{E S}_{p}$, then the domains of definition of $f$ and $g$ are the same, $V$ say, and $f_{1} \sim g_{1}$ in their partial-total factorizations $f=f_{1} p_{V}$ and $g=g_{1} p_{V}$.

Through the addition of symmetry event structures can represent a much richer class of categories than mere partial orders. The finite configurations of an event structure with symmetry can be extended by inclusion or rearranged bijectively under an isomorphism allowed by the symmetry. In this way an event structure with symmetry determines, in general, a category of finite configurations with maps those injections obtained by repeatedly composing the inclusions and allowed isomorphisms. By property (ii) in the definition of isomorphism family any such map factors uniquely as an isomorphism of the symmetry followed by an inclusion.

3) Constructions: We review from [10] important constructions in $\mathcal{E S}$.

\section{a) Products:}

Theorem 4. Let $A$ and $B$ be objects in $\mathcal{E S}$. Their product in $\mathcal{E S}$ is given by $\left(A \times B ; l_{A} \times l_{B}, r_{A} \times r_{B}: \widetilde{A} \times \widetilde{B} \rightarrow A \times B\right)$, based on the product $A \times B$ in $\mathcal{E}$, and sharing the same projections, $\pi_{1}: A \times B \rightarrow A$ and $\pi_{2}: A \times B \rightarrow B$. Let $f, f^{\prime}: C \rightarrow A$ and $g, g^{\prime}: C \rightarrow A$ in $\mathcal{E S}$. If $f \sim f^{\prime}$ and $g \sim g^{\prime}$, then $\langle f, g\rangle \sim\left\langle f^{\prime}, g^{\prime}\right\rangle$.

b) Simple parallel composition: The operation of simple parallel composition of event structures $A \| B$ which juxtaposes the two event structures $A$ and $B$-with a finite set of events consistent if its overlaps with $A$ and $B$ are consistent-and acts similarly on maps, extends to a functor on $\mathcal{E S}$. The symmetry on $A \| B$ is $\widetilde{A} \| \widetilde{B}$ with left and right maps $l_{A} \| l_{B}$ and $r_{A} \| r_{B}$.

c) Pseudo pullbacks: It will have great bearing on this paper that, while $\mathcal{E S}$ does not have equalizers or pullbacks in 
general, it does have pseudo equalizers and pseudo pullbacks. We give their definition and refer the reader to [10] for proofs.

Maps $f, g: B \rightarrow C$ have a pseudo equalizer $i: E \rightarrow B$, i.e. $f i \sim g i$ and for any map $i^{\prime}: E^{\prime} \rightarrow A$ such that $f i^{\prime} \sim g i^{\prime}$, there is a unique map $h: E^{\prime} \rightarrow E$ such that $i^{\prime}=i h$. The object $E$ can be described as having configurations those $x \in \mathcal{C}(B)$ for which $f x \stackrel{\varphi}{\cong}_{C} g x$, where $\varphi_{x}$ is the bijection induced by $x$; its isomorphism family is the restriction of that on $B$.

Maps $f: A \rightarrow C, g: B \rightarrow C$ have a pseudo pullback given as the pseudo equalizer of $f \pi_{1}, g \pi_{2}: A \times B \rightarrow C$. We summarize its properties: The pseudo pullback comprises an object $D$ and maps $\pi_{1}: D \rightarrow A$ and $\pi_{2}: D \rightarrow B$ such that $f \pi_{1} \sim g \pi_{2}$ which satisfies the further property that for any object $D^{\prime}$ and maps $p_{1}: D^{\prime} \rightarrow A$ and $p_{2}: D^{\prime} \rightarrow B$ such that $f p_{1} \sim g p_{2}$, there is a unique map $h: D^{\prime} \rightarrow D$ such that $p_{1}=\pi_{1} h$ and $p_{2}=\pi_{2} h$. The pseudo pullback is defined up to isomorphism, and sometimes written $A \times_{C} B$.

Concretely, a configuration in $\mathcal{C}\left(A \times_{C} B\right)$ corresponds to a a triple

$$
x, f x \stackrel{\theta}{=}_{C} g y, y
$$

where the composite $x \cong f x \stackrel{\theta}{=}_{C} g y \cong y$ is a secured bijection between $x \in \mathcal{C}(A)$ and $y \in \mathcal{C}(B)$ in the sense of Proposition 1 .

4) Homotopy: We remark that the category $\mathcal{E S}$ (and $\mathcal{E S}_{r}$ ) has the structure of a homotopy category. In particular it has path objects. From the pseudo pullback

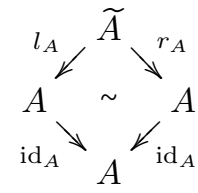

we recover $\widetilde{A}$, the symmetry on $A$, but as an object in $\mathcal{E S}$ itself equipped with symmetry $\widetilde{\widetilde{A}}$. This universal property is that associated with a path object generally written $A^{I}$, where $I$ stands for (a generalization of) the unit interval: asserting $\theta: x \cong_{A} y$, that a bijection between two configurations is in the isomorphism family of $A$, is analogous to specifying a path from $x$ to $y$. (There are also cylinder objects in $\mathcal{E S}$.)

Later, in defining the copycat strategy we shall make essential use of the fact that in $(A, \widetilde{A})$, an event structure with symmetry, the symmetry $\widetilde{A}$ itself possesses a symmetry $\widetilde{\widetilde{A}}$. Concretely, a configuration of $\widetilde{A}$ corresponds to an element of the isomorphism family $\theta: x \cong{ }_{A} y$ and a configuration of $\widetilde{\widetilde{A}}$ to a pair $\varphi_{1}, \varphi_{2}$ in a commuting square mediating between $\theta_{1}$ and $\theta_{2}$ in the isomorphism family of $A$ :

$$
\begin{array}{cll}
x_{1} & \stackrel{\theta_{1}}{\cong} & y_{1} \\
\varphi_{1} \text { ㄴ } A & & A^{\text {Il }} \varphi_{2} \\
x_{2} & \stackrel{\theta_{2}}{\cong} & y_{2}
\end{array}
$$

Lemma 5. Let $f: A \rightarrow B$ and $g: B \rightarrow C$ be maps in $\mathcal{E S}$ with pseudo pullback $P$ with maps $\pi_{1}: P \rightarrow A$ and $\pi_{2}: P \rightarrow B$. Then, $\widetilde{P}$, as an object in $\mathcal{E S}$, with maps $\widetilde{\pi}_{1}$ and $\widetilde{\pi}_{2}$ is a pseudo pullback of $\widetilde{f}$ and $\widetilde{g}$ in $\mathcal{E S}$.

\section{Adding polarity}

Games and strategies will be represented in terms of event structures where events are moves of Player or Opponent, signified by events carrying a polarity, + for Player and - for Opponent. Formally, an event structure with polarity comprises an event structure $A$ with a function $\operatorname{pol}_{A}: A \rightarrow\{+,-\}$ assigning a polarity to each event. Maps are assumed to preserve polarity.

The addition of polarity to an event structure $A$ means that the $\subseteq$-order on its finite configurations is now obtained as compositions $\left(\subseteq^{-} \cup \subseteq^{+}\right)^{+}$of two more fundamental orders, where for $x, y \in \mathcal{C}^{\infty}(A)$,

$$
\begin{aligned}
& x \subseteq^{-} y \text { iff } x \subseteq y \& \operatorname{pol}_{A}(y \backslash x) \subseteq\{-\}, \text { and } \\
& x \subseteq^{+} y \text { iff } x \subseteq y \& \operatorname{pol}_{A}(y \backslash x) \subseteq\{+\} .
\end{aligned}
$$

More surprisingly there is a new partial order, the Scott order, between configurations which is intimately related to copycat strategies. The Scott order $\subseteq_{A}$ is defined to be $\left(\beth^{-} \cup \subseteq^{+}\right)^{+}$. (We use $\beth^{-}$for the converse order to $\subseteq^{-}$.) The Scott order possesses a unique factorization: for $x, y \in \mathcal{C}^{\infty}(A)$,

$$
y \sqsubseteq_{A} x \Longleftrightarrow \exists ! z \in \mathcal{C}^{\infty}(A) . y \supseteq^{-} z \subseteq^{+} x
$$

-it is an easy exercise to show that $z$ is necessarily $x \cap y$. Not only do the configurations of copycat strategies of $A$ correspond to pairs of configurations in the Scott order, but also strategies in $A$ correspond to certain, simply-described, discrete fibrations over $\left(\mathcal{C}(A), \varsigma_{A}\right)$ - see [14] for the full story. Given this we can expect a variation on the Scott order to play a role in strategies in games with symmetry.

An event structure with polarity and symmetry (henceforth an e.p.s.) consists of $(E, \widetilde{E})$, an event structure with polarity also endowed with a symmetry $\widetilde{E}$. The categories $\mathcal{E P S}, \mathcal{E P S}$ r and $\mathcal{E P S}_{p}$ of such objects has maps preserving both symmetry and polarity, which are respectively total, rigid and partial. There are obvious forgetful functors to the respective categories in which polarity is ignored, and e.g. pseudo pullbacks are inherited from there. The inclusion functors $\mathcal{E P S}{ }_{r} \hookrightarrow \mathcal{E P S}$ and $\mathcal{E P S} \hookrightarrow \mathcal{E P S}_{p}$ inherit right adjoints from the situation without polarity, and again $\mathcal{E P S}$ and $\mathcal{E P S}_{p}$ are the Kleisli categories of the respective monads.

The addition of polarity and symmetry brings a new richness to the configurations of an event structure. The Scott order becomes a $S$ cott category, $S \operatorname{cott}(A)$, of an e.p.s. $A$, where now maps between configurations are obtained as compositions of $\beth^{-}, \subseteq^{+}$and the isomorphism family $\cong A$. Maps from $y$ to $x$ in $\operatorname{Scott}(A)$ have a unique factorization

$$
y \supseteq^{-} y_{0} \stackrel{\theta}{=}_{A} x_{0} \subseteq^{+} x .
$$

The unique factorization follows from property (ii) of isomorphism families and uniqueness of the factorization of the Scott order. A map $f: A \rightarrow B$ in $\mathcal{E P S}_{p}$ preserves $\supseteq^{-}, \subseteq^{+}$ and isomorphism families so extends directly to a functor $f: S \operatorname{cott}(A) \rightarrow S \operatorname{cott}(B)$ making $S \operatorname{cott}$ a functor to Cat. 


\section{GAMES WITH SYMMETRY}

A concurrent game with symmetry is represented by an e.p.s. A. A pre-strategy in $A$ is a total map $\sigma: S \rightarrow A$ in $\mathcal{E P S}$. A map between pre-strategies, from $\sigma: S \rightarrow A$ to $\sigma^{\prime}: S^{\prime} \rightarrow A$, is a map $f: S \rightarrow S^{\prime}$ in $\mathcal{E P S}$ such that

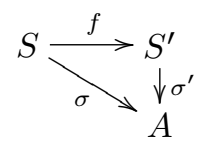

commutes. We say the two pre-strategies $\sigma$ and $\sigma^{\prime}$ are equivalent, and write $\sigma \simeq \sigma^{\prime}$, and sometimes $f: \sigma \simeq \sigma^{\prime}$, when there are maps $f$ from $\sigma$ to $\sigma^{\prime}$, and $g$ from $\sigma^{\prime}$ to $\sigma$ determining an equivalence $S \simeq S^{\prime}$, i.e. such that $g f \sim \operatorname{id}_{S}$ and $f g \sim \operatorname{id}_{S^{\prime}}$; isomorphism $\sigma \cong \sigma^{\prime}$ occurs when $g f=\operatorname{id}_{S}$ and $f g=\operatorname{id}_{S^{\prime}}$. A weak map from $\sigma$ to $\sigma^{\prime}$ is a map $f: S \rightarrow S^{\prime}$ such that the triangle above commutes up to $\sim$ and analogously say $\sigma$ and $\sigma^{\prime}$ are weakly equivalent when there are weak maps $f$ and $g$ making $S \simeq S^{\prime}$.

Simple parallel composition extends directly to a functor $A \| B$ on $A$ and $B$ in $\mathcal{E P S}$. The dual of an e.p.s. $A$, written $A^{\perp}$, has the same underlying event structure with symmetry but with a reversal of polarities.

Following Joyal [15], a pre-strategy from $A$ to $B$ is a pre-strategy in the game $A^{\perp} \| B$. In refining the notion of pre-strategy to that of strategy we shall follow the guiding principle of [8]: a strategy is a pre-strategy for which copycat is an identity w.r.t. composition. The next few sections make this precise and culminate in the definition of strategy between concurrent games with symmetry.

\section{A. Copycat}

Let $A$ be an e.p.s.. Configurations of $\mathrm{CC}_{A}$ will correspond to maps in $\operatorname{Scott}(A)$. Recall a map from $y$ to $x$ in $\operatorname{Scott}(A)$ can be put into a unique form

$$
y \beth^{-} y_{0} \stackrel{\theta}{\cong} A x_{0} \subseteq^{+} x .
$$

Define $q(x, \theta, y)$ to be the partial order with underlying set $x \| y=_{\text {def }}(\{1\} \times x) \cup(\{2\} \times y)$, causal dependency that inherited from $A^{\perp} \| A$ with additional causal dependencies

$$
\begin{aligned}
& \left\{\left(a_{1}, a_{2}\right) \in x_{0} \times y_{0} \mid \operatorname{pol}_{A}\left(a_{2}\right)=+\& \theta\left(a_{1}\right)=a_{2}\right\} \cup \\
& \left\{\left(a_{2}, a_{1}\right) \in y_{0} \times x_{0} \mid \operatorname{pol}_{A^{\perp}}\left(a_{1}\right)=+\& \theta\left(a_{1}\right)=a_{2}\right\} .
\end{aligned}
$$

That $q(x, \theta, y)$ is indeed a partial order follows as in [8]. The set of all such partial orders forms a rigid family $\mathcal{Q}$. We define the event structure of $C_{A}$ to be $\operatorname{Pr}(\mathcal{Q})$. Because Scott is a functor, the operation $C_{A}$ is functorial in $A$. We define the symmetry on $\mathrm{CC}_{A}$ as

$$
\mathrm{CC}_{A} \stackrel{\mathrm{CC}_{l_{A}}}{\longleftarrow} \mathrm{CC}_{\widetilde{A}} \stackrel{\mathrm{C}_{r_{A}}}{\longrightarrow} \mathrm{CC}_{A}
$$

Note, that in the construction of $\mathrm{CC}_{\widetilde{A}}$ we are using the fact that $\widetilde{A}$ possesses a symmetry $\widetilde{\widetilde{A}}$ - see Section II-B4. The map $\gamma_{A}: \mathbb{C C}_{A} \rightarrow A^{\perp} \| A$ takes a prime order to its maximum event.

Lemma 6. The construction $\left(\mathrm{CC}_{A}, \mathrm{CC}_{l_{A}}, \mathrm{CC}_{r_{A}}: \mathrm{CC}_{\widetilde{A}} \rightarrow \mathrm{CC}_{A}\right)$ is an e.p.s. and $\gamma_{A}$ a pre-strategy.
In future we shall overload $\mathrm{CC}_{A}$ and write $\mathrm{CC}_{A}$ for the e.p.s. of Lemma 6. From the definitions, $\widetilde{\mathbb{C}_{A}}=C_{\widetilde{A}}$.

\section{B. Composition}

Let $\sigma: S \rightarrow A^{\perp} \| B$ and $\tau: T \rightarrow B^{\perp} \| C$ be pre-strategies between games $A, B, C$. To define their composition first form the pseudo pullback

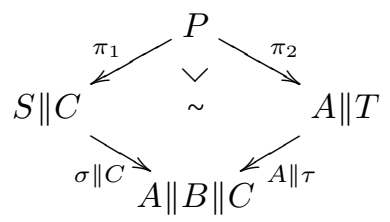

of the maps on the underlying event structures with symmetry, ignoring polarities, viz. $\sigma: S \rightarrow A \| B$ and $\tau: T \rightarrow B \| C$. There is an obvious partial map of event structures $A\|B\| C \rightarrow A \| C$ undefined on $B$ and acting as identity on $A$ and $C$. The partial maps from $P$ to $A \| C$, given by following the diagram either way round the pseudo pullback,

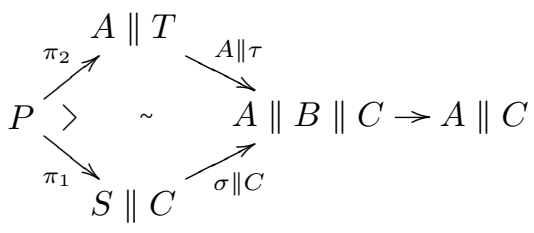

are defined on a common subset $V \subseteq P$-Proposition 3. Forming the partial-total factorization of either map (for preciseness take the left/lowest) we obtain

$$
P \rightarrow P \downarrow V \rightarrow A \| C .
$$

The resulting total map gives us the composition

$$
\tau \odot \sigma: T \odot S=_{\text {def }} P \downarrow V \rightarrow A^{\perp} \| C
$$

once we reinstate polarities to make $\tau \odot \sigma$ a map in $\mathcal{E P S}$. The projection operation $P \downarrow V$ hides all events not visible in $A$ and $C$.

From Lemma 5, regarding e.g. $\widetilde{T}$ as itself an e.p.s., it follows that $\widetilde{T \odot S} \cong \widetilde{T} \odot \widetilde{S}$ and $\widetilde{\tau \odot \sigma} \cong \widetilde{\tau} \odot \widetilde{\sigma}$.

\section{Strategies}

We are interested in necessary and sufficient conditions on a pre-strategy $\sigma: S \rightarrow A$ to ensure $\sigma \simeq \gamma_{A} \odot \sigma$. As we shall see $\sigma$ should be equivalent to a pre-strategy which is strongreceptive, innocent and saturated. (If a pre-strategy $\sigma: S \rightarrow$ $A^{\perp} \| B$ satisfies these conditions, then $\sigma \simeq \gamma_{B} \odot \sigma \odot \gamma_{A}$.)

1) Necessity: We show that for any pre-strategy $\sigma: S \rightarrow A$ the pre-strategy $\gamma_{A} \odot \sigma$ is necessarily strong-receptive, innocent and saturated.

A pre-strategy $\sigma$ is receptive iff for all $x \in \mathcal{C}(S)$, $\sigma x \stackrel{a}{\subset} \& \operatorname{pol}_{A}(a)=-$ implies $\exists ! s \in S . x \stackrel{s}{\subset} \& \sigma(s)=a$. The pre-strategy $\sigma: S \rightarrow A$, as a map in $\mathcal{E P S}$ preserves symmetry, so is associated with a map $\widetilde{\sigma}: \widetilde{S} \rightarrow \widetilde{A}$. Say $\sigma$ is strongreceptive if $\widetilde{\sigma}$, and so also $\sigma$, is receptive.

A pre-strategy $\sigma$ is innocent when $s \rightarrow s^{\prime}$ and $(\operatorname{pol}(s)=+$ or $\left.\operatorname{pol}\left(s^{\prime}\right)=-\right)$ implies $\sigma(s) \rightarrow \sigma\left(s^{\prime}\right)$. 
To specify when a pre-strategy $\sigma: S \rightarrow A$ is saturated we need some background. Form the pseudo pullback



The operation taking $\sigma$ to its saturation $\pi_{2}$ is part of a monad on pre-strategies in $A$. Clearly



commutes. Hence there is a unique map $\eta: S \rightarrow S \times_{A} A$ such that $\pi_{1} \eta=\operatorname{id}_{S}$ and $\pi_{2} \eta=\sigma$-thus $\eta$ is a map from $\sigma$ to $\pi_{2}$. We say $\sigma$ is saturated when $\eta$ is part of an equivalence $\sigma \simeq \pi_{2}$, i.e. there is a map act $: S \times_{A} A \rightarrow S$ such that



commutes and $a c t \eta \sim \operatorname{id}_{S}$ with $\eta a c t \sim \operatorname{id}_{S \times_{A} A}$. Concretely, a finite configuration of $S \times_{A} A$ can be identified with a pair $(x, \theta)$ where $x \in \mathcal{C}(S)$ and $\theta: \sigma x \cong_{A} y$. The action of act is to transport the configuration $x$ across $\theta$ it to a configuration $x^{\prime}={ }_{\operatorname{def}} \operatorname{act}(x, \theta)$ with $\varphi: x \cong_{S} x^{\prime}$ and $\widetilde{\sigma} \varphi=\theta$.

As an artifact of the definition of composition certain compositions are automatically saturated:

Proposition 7. . The composition $\tau \odot \sigma$ of pre-strategies $\sigma$ : $\varnothing \rightarrow B$ and $\tau: B \rightarrow C$ is saturated.

Proof. Adopt the notation of the diagram defining the composition $\tau \odot \sigma$ in Section III-B, with $A=\varnothing$. Let $u \in \mathcal{C}(T \odot S)$. Its down-closure $[u]$ is a configuration in the pseudo pullback $P$, and so corresponds to a secured bijection $x \| v \stackrel{\varphi}{\cong} y$ where $x \in \mathcal{C}(S), v \in \mathcal{C}(C)$ and $y \in \mathcal{C}(T)$. Because we define $\tau \odot \sigma$ to be got via the left way round the pseudo pullback square the configuration $u$ is sent to $v$ via $\tau \odot \sigma$. Consequently, given $\theta: v \cong_{C} v^{\prime}$ we can define $\operatorname{act}(u, \theta)$ to be that configuration $u^{\prime} \in \mathcal{C}(T \odot S)$ with down-closure $x\left\|v^{\prime} \stackrel{x \| \theta}{\cong} x\right\| v \stackrel{\varphi}{\cong} y$ in the pseudo pullback.

Lemma 8. $\gamma_{A}$ is strong-receptive, innocent and saturated.

Proof. The construction of $\mathrm{CC}_{A}$ directly ensures the innocence and receptivity of $\gamma_{A}$. The way symmetry of copycat is obtained from $C_{\widetilde{A}}$ makes $\widetilde{\gamma_{A}}$ equal $\gamma_{\widetilde{A}}$ so receptive, guaranteeing strong-receptivity of $\gamma_{A}$. To see $\gamma_{A}$ is saturated we require a map act: $\mathrm{CC}_{A} \times_{A \| A} A \| A \rightarrow \mathrm{CC}_{A}$. A configuration of $\mathrm{CC}_{A} \times{ }_{A \| A} A \| A$ corresponds to a configuration of $\mathrm{CC}_{A}$, so a map in $\operatorname{Scott}(A)$

$$
x \supseteq^{+} x_{0} \stackrel{\theta}{\cong} A y_{0} \subseteq^{-} y,
$$

and a configuration of $A \| A$, so a pair $v, w$, for which $\varphi_{1}: x \cong_{A}$ $v$ and $\varphi_{2}: y \cong_{A} w$. This data and the factorization properties in $\operatorname{Scott}(A)$ yield:

$\begin{array}{ccccccc}x & \supseteq^{+} & x_{0} & \stackrel{\theta}{\cong} & y_{0} & \subseteq^{-} & y \\ \varphi_{1}^{2 \|} A & & { }^{2 \|} A & & A^{\| 2} & & A^{\| 2} \varphi_{2} \\ v & \supseteq^{+} & v_{0} & \stackrel{\theta}{\prime}_{A} & w_{0} & \subseteq^{-} & w\end{array}$

We take the configuration of $\mathrm{CC}_{A}$ got via act to be that corresponding to



The map act together with $\eta: \mathrm{CC}_{A} \rightarrow \mathrm{CC}_{A} \times_{A \| A} A \| A$ establishes the equivalence needed for $\gamma_{A}$ to be saturated.

Lemma 9. $\gamma_{A} \odot \sigma$ is strong-receptive, innocent and saturated for any pre-strategy $\sigma$ in $A$.

Proof. The composition $\gamma_{A} \odot \sigma$ inherits innocence and receptivity directly from that of $\gamma_{A}$. Now $\overline{\gamma_{A} \odot \sigma} \cong \widetilde{\gamma_{A}} \odot \widetilde{\sigma} \cong \gamma_{\widetilde{A}} \odot \widetilde{\sigma}$ whence $\overline{\gamma_{A} \odot \sigma}$ inherits receptivity from that of $\gamma_{\widetilde{A}}$, making $\gamma_{A} \odot \sigma$ strong-receptive. The composition is saturated for general reasons-Proposition 7.

2) Sufficiency: We show the conditions strong-receptive, innocent and saturated are sufficient to ensure that a prestrategy $\sigma$ is equivalent to its composition with copycat $\gamma_{A} \odot \sigma$.

Lemma 10. Let $\sigma: S \rightarrow A$ be a pre-strategy. There is a map $I: S \rightarrow \mathbb{C}_{A} \odot S$ in $\mathcal{E P S}$, unique up to symmetry, such that $\sigma=\left(\gamma_{A} \odot \sigma\right) I$.

Proof. We sketch the existence part of the proof by describing how $I$ acts on configurations. Given $x \in \mathcal{C}(S)$, there is a secured bijection $x \| \sigma x \cong q\left(\sigma x, \mathrm{id}_{\sigma x}, \sigma x\right)$-the bijection is that given by $x \cong \sigma x$ between left components and $\mathrm{id}_{\sigma x}$ between the right. The secured bijection corresponds to a configuration $z$ of the pseudo pullback $(S \| A) \times_{A \| A} \mathbb{C C}_{A}$. The result of the projection operation is to hide all those events not above the right component in $A \| A$, so from $z$ yields a configuration of $\mathbb{C C}_{A} \odot S$ with image $\sigma x$ under $\gamma_{A} \odot \sigma$.

Lemma 11. If a pre-strategy $\sigma$ is strong-receptive, innocent and saturated, then $I: \sigma \simeq \gamma_{A} \odot \sigma$.

Proof. We require a map $K: \mathrm{CC}_{A} \odot S \rightarrow S$ that with $I$

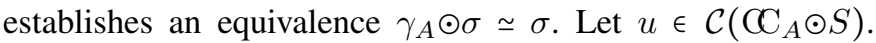
Its down-closure $[u]$ is a configuration in the pseudo pullback $(S \| A) \times_{A \| A}{C C_{A}}_{A}$ :

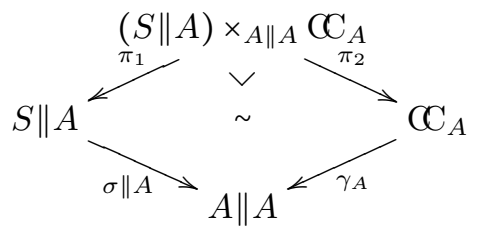

As a configuration of the pseudo pullback, $[u]$ corresponds to a triple, a configuration in $S \| A$ and a configuration in $\mathrm{CC}_{A}$, mediated by an element of the isomorphism family of $A \| A-$ see Section II-B3c. A configuration of $S \| A$ corresponds to a 
pair of configurations $x$ of $S$ and $w$ of $A$; a configuration of $\mathrm{CC}_{A}$ to configurations of $A$ in the relations $z_{1} \supseteq^{+} z_{1}^{\prime} \cong_{A} z_{2}^{\prime} \subseteq^{-}$ $z_{2}$; and the mediating element of the isomorphism family to a pair $\sigma x \cong_{A} z_{1}$ and $w \cong_{A} z_{2}$. From this data we obtain the composite map

$$
\sigma x \cong_{A} z_{1} \supseteq^{+} z_{1}^{\prime} \cong_{A} z_{2}^{\prime} \subseteq^{-} z_{2} \cong_{A} w
$$

in $\operatorname{Scott}(A)$. This factors uniquely into

$$
\sigma x \supseteq^{+} y_{1} \stackrel{\theta}{=}_{A} y_{2} \subseteq^{-} w .
$$

From innocence it follows (see Lemma 1 of [14]) that there is a unique $x_{1} \in \mathcal{C}(S)$ for which $x \underline{\supseteq}^{+} x_{1}$ and $\sigma x_{1}=y_{1}$. Now, the pair $x_{1}$ and $\theta: y_{1} \cong_{A} y_{2}$ can be identified with a configuration of $S \times{ }_{A} A$. Hence we can apply act to obtain $\operatorname{act}\left(x_{1}, \theta\right) \in \mathcal{C}(S)$ with $\sigma \operatorname{act}\left(x_{1}, \theta\right)=y_{2}$.

Define $p(u)=_{\text {def }} \operatorname{act}\left(x_{1}, \theta\right)$. By considering how it acts on isomorphism families, $p$ extends to a monotonic function

$$
\widetilde{p}: \mathcal{C}\left(\overline{\mathrm{C}_{A} \odot S}\right) \rightarrow \mathcal{C}(\widetilde{S})
$$

such that

$$
\forall u \in \mathcal{C}\left(\mathrm{CC}_{A} \odot S\right) . \widetilde{\sigma} \widetilde{p}(u) \subseteq^{-} \overline{\gamma_{A} \odot \sigma} u .
$$

Using Lemma 23 of [8] and the strong-receptivity of $\sigma$, we obtain a unique total map $K: \mathrm{CC}_{S} \odot S \rightarrow S$ such that $\forall u \in$ $\mathcal{C}\left(C_{A} \odot S\right) \cdot p(u) \subseteq^{-} K u$ and $\gamma_{A} \odot \sigma=\sigma K$. On checking $K I \sim$ $\operatorname{id}_{S}$ and $I K \sim \operatorname{id}_{C_{A} \odot S}$ we have the desired equivalence.

Define a strategy to be a pre-strategy which is strongreceptive, innocent and saturated. A weak strategy is a prestrategy which is weakly equivalent to $\gamma_{A} \odot \sigma$; so, directly from this definition, any weak strategy is weakly equivalent to a strategy. It is sometimes convenient to work with weak strategies (which need not be saturated) and then compose with copycat to obtain strategies. (We lack a direct characterization of weak strategies.)

Lemma 12. If two saturated pre-strategies are weakly equivalent they are equivalent. A fortiori, if two strategies are weakly equivalent they are equivalent.

Note that a strategy $\sigma: S \rightarrow A$ induces a fibration $\sigma$ : $S \operatorname{cott}(S) \rightarrow S \operatorname{cott}(A)$. In fact, $\supseteq^{-}$and $\subseteq^{+}$maps in $S \operatorname{cott}(A)$ have cartesian liftings again as $\supseteq^{-}$and $\subseteq^{+}$maps, respectively, in $S \operatorname{cott}(S)$ because $\sigma$ is receptive and innocent [14]—with strong-receptivity ensuring the appropriate uniqueness-while $\cong_{A}$ maps have cartesian liftings in $\cong_{S}$ because $\sigma$ is saturated.

Lemma 13. If $\sigma$ and $\tau$ are strategies, so is $\tau \odot \sigma$.

Proof. The composition inherits innocence and receptivity from $\sigma$ and $\tau$. Because $\widetilde{\tau \odot \sigma} \cong \widetilde{\tau} \odot \widetilde{\sigma}$ it also inherits strong receptivity. Its saturation obtains via Proposition 7 and the saturation of $\tau$.

3) A -bicategory of games with symmetry: Combining the above results, we do not quite obtain a bicategory but rather Strat, a "bicategory up to symmetry" in the following sense: - its objects are e.p.s.'s- the games;
- its arrows from $A$ to $B$ are strategies $\sigma: A \nrightarrow B$ related by maps of pre-strategies- $\operatorname{Strat}(A, B)$ is thus a category enriched with equivalence relations $\sim$;

- horizontal composition is given by composition of strategies $\odot$, which extends to functors $\operatorname{Strat}(B, C) \times \mathcal{S} \operatorname{trat}(A, B) \rightarrow$ $\operatorname{Strat}(A, C)$ via the universality of pseudo pullback;

- there is a natural isomorphism (derived from the universality of pseudo pullback) to express the associativity of composition, but only natural equivalences (derived from the equivalence of Lemma 11) for left and right identity laws;

- of the usual coherence axioms for bicategories, that for identity only commutes up to .

Because categories of event structures with symmetry are degenerate 2-categories, the above describes a special case of weak 3-category, which we call a -bicategory as it is morally a bicategory but where the axioms hold up to $\sim$. Weak strategies possess a similar weaker structure.

Strat is rich in structure. Observe the duality: a strategy $\sigma: A \rightarrow B$ corresponds to a strategy $\sigma^{\perp}: B^{\perp} \rightarrow A^{\perp}$. There is a bijective correspondence between strategies $A \| B \rightarrow C C$ and strategies $A \rightarrow\left(B^{\perp} \| C\right)$, making Strat monoidal closed, and in fact compact closed, in an extended sense.

To be more precise, and to relate to standard game semantics, we can quotient out the higher-dimensional structure to obtain a category. The category $\mathcal{S}$ trat/ $\simeq$ has e.p.s.'s as objects, and $\simeq$-equivalence classes of strategies $\sigma: S \rightarrow A^{\perp} \| B$ as morphisms from $A$ to $B$. The -bicategorical structure ensures that equivalence of strategies is preserved by composition, so we get a category $\mathcal{S}$ trat/ $\simeq$.

Proposition 14. The category $\mathcal{S}$ trat/ $\simeq$ is compact closed, with tensor product $\|$ and dual $(-)^{\perp}$.

The compact closed structure of $\mathcal{S}$ trat/ $\simeq$ is not so surprising: the category is defined in a similar fashion to Joyal's category of Conway games [15], which is compact closed as well. As compact closed categories, they are *-autonomous and hence models of Multiplicative Linear Logic (MLL) [16].

\section{ApPLICATIONS}

Once we have symmetry in games we can support a rich repertoire of (pseudo) monads on e.p.s.'s, and e.g. all the monads of [10] are undisturbed by the presence of polarity. Monads to support copying w.r.t. maps of e.p.s.'s can often translate to monads w.r.t. strategies, and so by duality also to comonads w.r.t. strategies. Following Girard's work on linear logic [17], this opens up the possibility of modelling programming languages that are not resource-sensitive, in that (copies of) the same resource can be used multiple times. We describe, in particular, how AJM games [9] and $\mathrm{HO}$ games [18] generalize and can be recovered from concurrent games. The (co)monads involved rely pivotally on the presence of symmetry. Their structure lifts from simple structural maps.

\section{A. Maps as strategies}

A structural pair $f=\left(f^{L}, f^{R}\right): A \rightarrow B$ comprises - $f^{L}: A \rightarrow B$, a total map of e.p.s.'s, as left component, and 
- $f^{R}: B \rightarrow A$, an injective, partial map of event structures, not necessarily preserving symmetry, as right component, such that $f^{R} \circ f^{L}=i d_{A}$. (Such pairs correspond to Kahn and Plotkin's rigid embeddings if we ignore symmetry.)

A structural pair $f: A \rightarrow B$, lifts to a strategy $\bar{f}: S(f) \rightarrow$ $A^{\perp} \| B$, obtained via a rigid family $\mathcal{Q}$. Whenever

$$
x \supseteq^{+} x^{\prime} \stackrel{f^{L}}{\mapsto} f^{L} x^{\prime} \stackrel{\theta}{\cong}_{B} y^{\prime} \subseteq^{-} y,
$$

with $x, x^{\prime} \in \mathcal{C}(A)$ and $y, y^{\prime} \in \mathcal{C}(B)$, define a typical $q(x, \theta, y) \in$ $\mathcal{Q}$ to have underlying set $x \| y$, and causal dependency that inherited from $A^{\perp} \| B$ with additional causal dependencies

$$
\begin{aligned}
& \left\{\left(a, \theta\left(f^{L} a\right)\right) \in x^{\prime} \times y^{\prime} \mid \operatorname{pol}_{A^{\perp}}(a)=-\right\} \cup \\
& \left\{\left(\theta\left(f^{L} a\right), a\right) \in y^{\prime} \times x^{\prime} \mid \operatorname{pol}_{A^{\perp}}(a)=+\right\} .
\end{aligned}
$$

The event structure $S(f)$ is then defined as $\operatorname{Pr}(\mathcal{Q})$, and the strategy-as-map $\bar{f}: S(f) \rightarrow A^{\perp} \| B$ by $\bar{f}(q)=a$, where $a$ is the top element of the prime $q \in S(f)$. Elements of the isomorphism family of $S(f)$ correspond to isomorphisms

$$
\begin{aligned}
& x_{1} \quad \supseteq^{+} \quad x_{1}^{\prime} \quad \stackrel{f}{\mapsto} f x_{1}^{\prime} \stackrel{\stackrel{\theta}{\cong}}{\cong_{B}} \quad y_{1}^{\prime} \quad \subseteq^{-} \quad y_{1} \\
& \varphi_{1}{ }^{211} A \quad \varphi_{2}{ }^{211} A \quad f \varphi_{2}{ }^{211} B \quad \varphi_{3}{ }^{211} B \quad \varphi_{4}{ }^{2 \|}{ }_{B} \\
& x_{2} \stackrel{\supseteq}{ }^{+} \quad x_{2}^{\prime} \quad \stackrel{f}{\mapsto} \quad f x_{2}^{\prime} \stackrel{\stackrel{\theta}{\cong}}{\cong_{B}} \quad y_{2}^{\prime} \quad \subseteq^{-} \quad y_{2} .
\end{aligned}
$$

This induces an isomorphism family because $f^{L}$ preserves symmetry, which by construction is preserved by $\bar{f}$. Additionally, one can check that $\bar{f}$ is strong-receptive, innocent and saturated, so a strategy. Note that this lifting operation also preserves equivalence: if $f \sim g$ then it is easy to show that $\bar{f}$ and $\bar{g}$ are weakly equivalent, so equivalent by Lemma 12 .

The motivation for this construction is the following lemma, relating composition of maps to that by their lifts as strategies.

Lemma 15. Let $\sigma: T \rightarrow A$ be a strategy and $f: A \rightarrow B$ be a structural pair. Then the pre-strategy $f^{L} \circ \sigma: T \rightarrow B$ is weakly equivalent to the strategy $\bar{f} \odot \sigma: S(f) \odot T \rightarrow B$.

We also need to examine the composition of a strategy with the dual of one lifted from a structural pair. A right map $f^{R}: B \rightarrow A$ of a structural pair does not necessarily preserve symmetry, but it does preserve a sub-symmetry, in the sense that the set of isomorphisms $x \stackrel{\theta}{\cong}$ 至 $y$, such that $f^{R} x \stackrel{f^{R} \theta}{\cong} A f^{R} y$, forms an isomorphism family. Then $A$ can be restricted to make $f^{R}$ a total map preserving symmetry. Write $\left(A \uparrow f^{R}\right)$ for the event structure with events those on which $f^{R}$ is defined and with isomorphism family that part preserved by $f^{R}$. Obviously, $f^{R}:\left(A \uparrow f^{R}\right) \rightarrow B$ is a total map preserving symmetry.

Lemma 16. Let $\sigma: T \rightarrow A$ be a strategy and $f: B \rightarrow A$ a structural pair. Then $f^{R} \circ \sigma$ preserves a sub-symmetry, and $f^{R} \circ \sigma:\left(T \vdash f^{R} \circ \sigma\right) \rightarrow B$ is a strategy equivalent to the strategy $(\bar{f})^{\perp} \odot \sigma: S(f) \odot T \rightarrow B$.

\section{B. AJM games}

We have not assumed that games are polarized, i.e. that initial moves share the same polarity, a condition imposed in most presentations of games. Non-polarized games are useful because they permit an account of negation as just polarityreversal, and hence model directly the involutive negation of Classical Linear Logic. In contrast, polarized games lose involutive negation and are restricted to modelling "polarized" logics, such as Intuitionistic Linear Logic (ILL). Through concurrent games we can give a concurrent version of the construction in [12] of a non-polarized adaptation of AJM games [9] to model classical linear logic.

A categorical model of Multiplicative Exponential Linear Logic (MELL) is a $*$-autonomous category $(\mathcal{C}, \otimes)$ (such as $\mathcal{S}$ trat/ $\simeq$ with a linear exponential comonad, i.e. a monoidal comonad $(!, \epsilon, \delta, m)$ with monoidal natural transformations $e_{A}: ! A \rightarrow 1$ and $d_{A}: ! A \rightarrow ! A \otimes ! A$ such that each $\left(! A, e_{A}, d_{A}\right)$ is a commutative comonoid, $e_{A}$ and $d_{A}$ are coalgebra maps and any coalgebra map between free coalgebras is also a comonoid morphism. We aim to build this structure

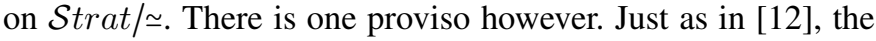
absence of polarization means that the naturality of weakening $e_{A}: ! A \rightarrow I$ will be missing, so we model classical linear logic in the sense of [12] and not quite MELL. If we restrict to negatively polarized games the naturality of $e_{A}$ is recovered at the cost of self-duality, yielding a model of ILL.

From a game $A$, we form the game ! $A$ comprising $\omega$ similar copies of $A$. Its events are pairs $(i, a)$ where $a \in A$ and $i \in \omega$, with causal dependency

$$
\left(i_{1}, a_{1}\right) \leq\left(i_{2}, a_{2}\right) \Leftrightarrow i_{1}=i_{2} \& a_{1} \leq_{A} a_{2}
$$

and consistency relation

$$
\operatorname{Con}_{! A}=\bigcup_{i \in I}\{i\} \times X_{i},
$$

where $I$ is a finite subset of $\omega$, and for each $i \in I, X_{i} \in \operatorname{Con}_{A}$. Polarity is inherited directly from $A$. We describe its symmetry as an isomorphism family. If $x, y \in \mathcal{C}(! A)$, we have $x \stackrel{\theta}{\cong} ! A$ if, writing $x=\bigcup_{i \in I}\{i\} \times x_{i}$ and $y=\bigcup_{j \in J}\{j\} \times y_{j}$ with each $x_{i}$ and $y_{j}$ nonempty, there is a bijection $\pi: I \cong J$ so $x_{i} \stackrel{\vartheta_{i}}{=} y_{\pi(i)}$, for each $i \in I$, where $\theta((i, a))=\left(\pi(i), \theta_{i}(a)\right)$ for all $(i, a) \in x$.

The construction extends to a functor on $\mathcal{E P S}$ : if $f: A \rightarrow$ $B$ then $! f: ! A \rightarrow ! B$ sends $(i, a)$ to $(i, f(a))$.defined. It is convenient to investigate the monad/monoid structure of ! in $\mathcal{E P S}$ first, their duals will be eventually deduced by duality in Strat. We have the following in $\mathcal{E P S}$ :

$$
\begin{aligned}
& \eta_{A}: A \rightarrow ! A \\
& \mu_{A}: ! ! A \rightarrow ! A \\
& q_{A, B} \quad: \quad !(A \| B) \rightarrow ! A \| ! B \\
& e_{A}: 1 \rightarrow ! A \\
& m_{A}: \text { : } A \| ! A \rightarrow ! A
\end{aligned}
$$

where $\eta_{A}$ sends any event $a$ to $(0, a) ; \mu_{A}$ tracks an arbitrary bijection between $\omega \times \omega$ and $\omega ; q_{A, B}$ is the obvious distribution map; $e_{A}$ is the empty map; and $m_{A}$ tracks an arbitrary bijection between $\omega+\omega$ and $\omega$. All these maps are natural in their parameters in the category $\mathcal{E P S}$. In particular, $(!, \eta, \mu)$ forms a monad on $\mathcal{E P S}$.

The functors $\|$ and ! on $\mathcal{E P S}$ extend to functors on strategies written $\|^{\mathcal{S}}$ and $!^{\mathcal{S}}$ for disambiguation: given strategies $\sigma_{1}$ : $S_{1} \rightarrow A_{1}^{\perp} \| B_{1}$ and $\sigma_{2}: S_{2} \rightarrow A_{2}^{\perp} \| B_{2}$, we have $\sigma_{1} \|^{\mathcal{S}}$ $\sigma_{2}: S_{1}\left\|S_{2} \rightarrow\left(A_{1} \| A_{2}\right)^{\perp}\right\|\left(B_{1} \| B_{2}\right)$ given by the 
obvious map of $\mathcal{E P S}$. To define $!^{\mathcal{S}} \sigma$, the obvious choice is the composition $q_{A_{1}^{\perp} B_{1}} \circ ! \sigma: ! S \rightarrow ! A_{1}^{\perp} \| ! B_{1}$, which we must then compose with copycat to obtain a strategy. These yield a bifunctor $\|^{\mathcal{S}}$ and a functor $!^{\mathcal{S}}$ on strategies, using the fact that ! and || preserve pseudo-pullbacks. By the tools of the previous section, the natural transformations above, apart from $e_{A}$, become natural transformations as strategies:

Lemma 17. The strategies $\overline{\epsilon_{A}}, \overline{\delta_{A}}, \overline{d_{A}}$ and $\overline{q_{A, B}}$ are natural in $A, B$ in the category $\mathcal{S}$ trat/ $\simeq$.

Proof. Each of these maps $f$ has an adjoint $f^{\bullet}$ making $\left(f, f^{\bullet}\right)$ a structural pair. By Lemmas 15 and 16 the compositions involved in the naturality squares can be computed by simple composition of maps, and the equivalences then amount to elementary verifications.

The monoid and monad laws follow from those in $\mathcal{E P S}$, since the strategies have been lifted from there. The fact that algebra morphisms between free algebras $\left(! A, \mu_{A}\right)$ satisfy the laws needed to be morphisms of monoids follows from a simple diagram chase, using that $\mu_{A}$ is an invertible monoid morphism and that this property remains true of $\overline{\mu_{A}}$.

We have established that the natural transformations above define the dual of a linear exponential comonad, short of the naturality of $e_{A}$. By self-duality of $\mathcal{S}$ trat/ $\simeq$, we therefore have:

Theorem 18. The category Strat/ $\simeq$ with ! is a model of classical linear logic in the sense of [12].

\section{HO games and HO-innocence}

We now apply concurrent games with symmetry to construct a concurrent generalization of the "plays with pointers" of HO games. Among the strategies for concurrent games "with pointers", we isolate the $\mathrm{HO}$-innocent strategies, that extend standard $\mathrm{HO}$ innocent strategies.

An e.p.s. $A$ is negative when all its minimal events have negative polarity, and well-threaded iff $[a]$ has one minimal event for any $a \in A$. A strategy $\sigma: S \rightarrow A$ is negative or well-threaded when $S$ is.

1) The linear category: We start by introducing a linear category. Its objects are negative well-threaded e.p.s.'s and a morphism from $A$ to $B$ is a negative well-threaded strategy $\sigma: S \rightarrow A^{\perp} \| B$. Well-threadedness is analogous to the condition of single-threadedness in $\mathrm{HO}$ games, ensuring there that strategies are comonoid morphisms [19] and hence form a cartesian closed category-it will serve a similar purpose here. These conditions are preserved by composition, and satisfied by copycat. Once quotiented, we obtain a category $\mathcal{N} e g$.

This category inherits a monoidal structure from $\mathcal{S}$ trat/ . It does not inherit its closed structure, because if $A$ and $B$ are both negative, then generally $A^{\perp} \| B$ is not. Closedness can nonetheless be recovered with the game $A \multimap B$ having:

- events, those of $\left(\|_{b \in \min (B)} A^{\perp}\right) \| B$ where $\min (B)$ denotes the set of minimal events of $B$;

- causal dependency, that of $\left(\|_{b \in \min (B)} A^{\perp}\right) \| B$ enriched with $\{((2, b),(1,(b, a))) \mid a \in A \& b \in \min (B)\}-$ in other words, all the events in each copy of $A$ depend on the corresponding minimal event of $B$;
- consistency, with $X=\left(\|_{b \in \min (B)} X_{b}\right) \| X_{2} \in \operatorname{Con}_{A \multimap B}$ iff $X_{2} \in \operatorname{Con}_{B}, \cup_{b \in \min (B)} X_{b} \in \operatorname{Con}_{A}$ and for all distinct $b_{1}, b_{2} \in \min (B), X_{b_{1}} \cap X_{b_{2}}=\varnothing$, i.e. the components on $A$ form a partition of a consistent set.

- isomorphism family, comprising $\theta: x \cong y$ for which there are isomorphisms $\theta_{1}$ and $\theta_{2}$ on $A^{\perp}$ and $B$ respectively, such that for all $(2, b) \in x, \theta((2, b))=\left(2, \theta_{2}(b)\right)$ and for all $(1,(b, a)) \in$ $x, \theta((1,(b, a)))=\left(1,\left(\theta_{2}(b), \theta_{1}(a)\right)\right)$.

Lemma 19. The category $(\mathcal{N e g}, \|, \multimap)$ is symmetric monoidal closed.

Proof. If $\sigma: S \rightarrow A^{\perp}\left\|B^{\perp}\right\| C$ is negative and well-threaded, then for each $s \in S$ there is a unique minimal $s^{\prime} \in S$ necessarily mapping to $C$ on which $s$ depends, making unambiguous the copy of $B$ in $B \multimap C$ to which $s$ should be sent.

2) Concurrent games "with pointers": Arenas are negative countable forests $(A, \leq)$ without symmetry. They are closed by $\|$ and support the additive implication of [20], written $A \boxplus B$ and defined as for $A \multimap B$ without the consistency relation.

From an arena $A$ we define a game $? A$ understood as " $A$ with pointers". Its definition requires the notion of an exponential slice: a slice for an event $a$ is a function $\alpha:[a] \rightarrow \omega$, so gives a copy index for each event on which $a$ depends. For two slices $\alpha:[a] \rightarrow \omega$ and $\alpha^{\prime}:\left[a^{\prime}\right] \rightarrow \omega$, we write $\alpha$ $\alpha^{\prime}$ when $a \leq a^{\prime}$ and $\alpha(b)=\alpha^{\prime}(b)$ for all $b \leq a$. The game ? $A$ has: - events, pairs $(\alpha, a)$ where $\alpha:[a] \rightarrow \omega$;

- causal dependency, $(\alpha, a) \leq\left(\alpha^{\prime}, a^{\prime}\right)$ iff $\alpha \sqsubseteq \alpha^{\prime}$,

- consistency, all finite subsets of $? A$.

- isomorphism family, bijections $\theta: x \cong y$ such that for all $(\alpha, a) \in x$ there is $\alpha^{\prime}:[a] \rightarrow \omega$ with $\theta(\alpha, a)=\left(\alpha^{\prime}, a\right)$.

To convey the similarity with plays with pointers of $\mathrm{HO}$ games, we could say that $(\alpha, a)$ "points to" $\left(\alpha^{\prime}, a^{\prime}\right)$ when $\alpha^{\prime}$ 드 $\alpha$ and $a^{\prime} \rightarrow a$. However $?$ A leaves duplicated events causally unrelated whereas they would appear in some chronological order in a play with pointers. Configurations of $? A$ are more closely related to the thick subtrees of Boudes [21].

Notice the isomorphism $?(A \| B) \cong ? A \| ? B$. There is also an equivalence $?(A \multimap B) \simeq ? A \multimap ? B$, sending events of $A$ enabled by distincts events of $P B$ to distincts components of $? A$. We are interested in the objects of $\mathcal{N} e g$ of the form $? A$. A first remark is that they are comonoids; indeed we have maps of event structures $m_{A}: ? A^{\perp} \| ? A^{\perp} \rightarrow ? A^{\perp}$ and $e_{A}$ : $1 \rightarrow ? A^{\perp}$ satisfying monoid laws up to symmetry, and the lifting operation yields negative well-threaded strategies $\bar{m}_{A}{ }^{\perp}$ :

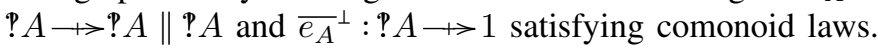
Analogously to standard $\mathrm{HO}$ games, we have the following.

Lemma 20. Negative well-threaded strategies from ? A to ? B are comonoid morphisms in $\mathcal{N}$ eg.

Proof. By well-threadedness and saturation.

It follows that the subcategory of $\mathcal{N} e g$ with objects of the form ? $A$ is cartesian closed.

3) Concurrent $\mathrm{HO}$ games: The category of concurrent $\mathrm{HO}$ games will have arenas as objects. Let us call sequential arenas the standard arenas of $\mathrm{HO}$ games (considered here to be 
alternating forests, as in [13] but without the Question/Answer labeling). Sequential arenas are obviously arenas in our sense, and our constructions $\|$ and $\rightarrow$ coincide with the familiar constructions $\times$ and $\Rightarrow$ on sequential arenas.

A $H O$-strategy from an arena $A$ to an arena $B$ is a negative, well-threaded strategy $\sigma: S \rightarrow ?(A-\boxplus B)$. To compose these, we use the following lemma:

Lemma 21. There is a bijection (up to equivalence) between


from $?$ A to $? B$ in $\mathcal{N}$ eg.

Proof. By the equivalence $?(A \multimap B) \simeq ? A \multimap ? B$ and the closed structure of $\mathcal{N} e g$.

Therefore they can be composed in $\mathcal{N} e g$; this yields a category HOStrat. By $?(A-B) \simeq ? A \multimap ? B$ again, $\mathcal{H O S}$ trat inherits the monoidal closure of $\mathcal{N} e g$. Moreover games of the form $? A$ are comonoids for $\|$ in $\mathcal{N} e g$, so by the

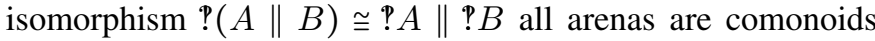
for $\|$ in $\mathcal{H O S}$ trat and HO-strategies are comonoid morphisms by Lemma 20; it follows that $\mathcal{H O S t r a t}$ is cartesian closed.

4) HO-innocent strategies: Finally, we isolate a subcategory of $\mathcal{H O S t r a t}$ isomorphic to the usual category of sequential arenas and innocent strategies. A HO-strategy $\sigma: S \rightarrow ? A$ on an arena $A$ is HO-innocent if for any $s \in S$, if $s_{1}, s_{2} \in[s]$ have negative polarity then $s_{1} \leq s_{2}$ or $s_{2} \leq s_{1}$. In other words there are no negative concurrent events in a prime configuration $[s]$, so Player never waits for two concurrent Opponent events to play. In particular, if Opponent opens two independent copies of a subgame they must appear in distinct prime configurations of $S$, so they are treated independently.

Proposition 22. Arenas and HO-innocent strategies form a sub-CCC, say IHOStrat, of $\mathcal{H O S}$ Strat.

Proof. Copycat is HO-innocent on arenas, and HO-innocence is preserved by all operations on strategies. Preservation of HO-innocence by composition follows from a simple analysis of causality in the pseudo-pullback defining composition.

The category IHOStrat deserves to be explored further. But for our purpose here it is still too general: its strategies are still nondeterministic and concurrent. We therefore impose two further conditions:

An HO-innocent strategy $\sigma: S \rightarrow ? A$ is deterministic iff whenever $x \stackrel{\varphi}{\cong} y$ and $x \stackrel{+}{\complement} x^{\prime}$ and $y-\subset y^{\prime}$, then $x^{\prime} \subseteq x^{\prime \prime}$ and $y^{\prime} \subseteq y^{\prime \prime}$ for some $x^{\prime \prime}, y^{\prime \prime} \in \mathcal{C}(S)$ with $\varphi^{\prime}: x^{\prime \prime} \cong_{S} y^{\prime \prime}$ such that $\varphi \subseteq \varphi^{\prime}$-this is a generalization of the notion of deterministic concurrent strategies [22] in the presence of symmetry. It is sequential iff it never starts parallel threads, i.e. for any $s \in S$ such that $[s]$ extends by distinct positive events $s_{1}, s_{2}$, then $[s] \cup\left\{s_{1}, s_{2}\right\} \notin \operatorname{Con}_{S}$. Both conditions are stable under composition along with HO-innocence, and are satisfied by copycat on games of the form ? $A$, for any sequential arena $A$.

Theorem 23. The subcategory of IHOStrat having sequential arenas as objects and deterministic sequential $\mathrm{HO}$ innocent strategies as morphisms is isomorphic to the usual category of arenas and innocent strategies.
Proof. The conditions on strategies and arenas force their prime configurations $[s]$ to be alternating chains $s_{1}^{-} \rightarrow s_{2}^{+} \rightarrow$ $s_{3}^{-} \rightarrow \ldots \rightarrow s_{n}$. These chains correspond exactly to $P$-views, so the set of such chains is a set of $P$-views that is $O$-branching by determinism and sequentiality. This correspondence is bijective (up to equivalence), and preserves composition.

Concurrent games with symmetry have provided a new construction of the usual category of arenas with HO-innocent strategies and a new proof that HO-innocent strategies are stable under composition, within a generalization to concurrency and nondeterminism. In the past, providing a notion of nondeterministic HO-innocence has proved challenging [19].

Acknowledgements Thanks to Martin Hyland for advice and encouragement and to the ERC for Advanced Grant ECSYM.

\section{REFERENCES}

[1] D. R. Ghica and A. S. Murawski, "Angelic semantics of fine-grained concurrency," in FoSSaCS, ser. Lecture Notes in Computer Science, I. Walukiewicz, Ed., vol. 2987. Springer, 2004, pp. 211-225.

[2] J. Laird, "A game semantics of the asynchronous pi-calculus," in CONCUR, ser. Lecture Notes in Computer Science, M. Abadi and L. de Alfaro, Eds., vol. 3653. Springer, 2005, pp. 51-65.

[3] D. R. Ghica and A. S. Murawski, "Compositional model extraction for higher-order concurrent programs," in TACAS, ser. Lecture Notes in Computer Science, H. Hermanns and J. Palsberg, Eds., vol. 3920. Springer, 2006, pp. 303-317.

[4] S. Abramsky and P.-A. Melliès, "Concurrent games and full completeness," in LICS '99. IEEE Computer Society, 1999.

[5] P.-A. Melliès, "Asynchronous games 2: The true concurrency of innocence," Theor. Comput. Sci., vol. 358, no. 2-3, pp. 200-228, 2006.

[6] P.-A. Melliès and S. Mimram, "Asynchronous games: Innocence without alternation," in CONCUR, ser. Lecture Notes in Computer Science, L. Caires and V. T. Vasconcelos, Eds., vol. 4703. Springer, 2007, pp. 395-411.

[7] C. Faggian and M. Piccolo, "Partial orders, event structures and linear strategies," in TLCA '09, ser. LNCS, vol. 5608. Springer, 2009.

[8] S. Rideau and G. Winskel, "Concurrent strategies," in LICS 2011. IEEE Computer Society, 2011.

[9] S. Abramsky, R. Jagadeesan, and P. Malacaria, "Full abstraction for PCF,' Inf. Comput. 163(2): 409-470, 2000.

[10] G. Winskel, "Event structures with symmetry," Electr. Notes Theor. Comput. Sci. 172: 611-652, 2007.

[11] A. Joyal, M. Nielsen, and G. Winskel, "Bisimulation from open maps," Inf. Comput., vol. 127, no. 2, pp. 164-185, 1996.

[12] P. Baillot, V. Danos, T. Ehrhard, and L. Regnier, "Believe it or not, ajm's games model is a model of classical linear logic," in LICS. IEEE Computer Society, 1997, pp. 68-75.

[13] J. M. E. Hyland and C.-H. L. Ong, "On full abstraction for PCF: I, II, and III." Inf. Comput. 163(2): 285-408, 2000.

[14] G. Winskel, "Strategies as profunctors," in FOSSACS 2013. LNCS, Springer, 2013.

[15] A. Joyal, "Remarques sur la théorie des jeux à deux personnes," Gazette des sciences mathématiques du Québec, 1(4), 1997.

[16] P. Melliès, "Categorical semantics of linear logic," Panoramas et Syntheses, Société Mathématique de France, 2008.

[17] J.-Y. Girard, "Linear logic," Theor. Comput. Sci., vol. 50, pp. 1-102, 1987.

[18] M. Hyland and L. Ong, "On full abstraction for pcf," Information and Computation, vol. 163, pp. 285-408, 2000.

[19] R. Harmer, Games and full abstraction for non-deterministic languages, 1999, phD thesis, University of London.

[20] R. Harmer, M. Hyland, and P.-A. Melliès, "Categorical combinatorics for innocent strategies," in LICS '07. IEEE Computer Society, 2007.

[21] P. Boudes, "Thick subtrees, games and experiments," in TLCA, ser. Lecture Notes in Computer Science, P.-L. Curien, Ed., vol. 5608. Springer, 2009, pp. 65-79.

[22] G. Winskel, "Deterministic concurrent strategies," Formal Asp. Comput., vol. 24 , no. 4-6, pp. 647-660, 2012. 


\section{APPENDIX}

Assume a category with pullbacks. A pair of maps $l, r$ : $R \rightarrow E$ forms an equivalence relation provided it is

Jointly monic: for all maps $x, y: D \rightarrow R$, if $l x=l y$ and $r x=r y$, then $x=y$;

Reflexive: there is a (necessarily unique) map $\rho$ such that

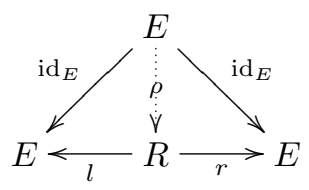

commutes;

Symmetric: there is a (necessarily unique) map $\sigma$ such that

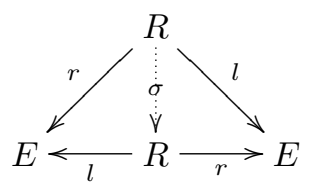

commutes;

Transitive: there is a (necessarily unique) map $\tau$ such that

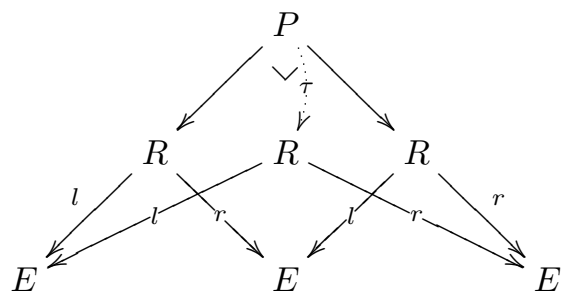

commutes. 